\title{
A CONNECTEDNESS PROPERTY OF MAXIMAL MONOTONE OPERATORS AND ITS APPLICATION TO APPROXIMATION THEORY
}

\author{
LIBOR VESELÝ
}

(Communicated by Palle E. T. Jorgensen)

\begin{abstract}
We prove a connectedness property of a general maximal monotone operator on a Hilbert space. As a consequence we easily obtain the characterization of components of the set of discontinuity points for metric projections of closed sets in Hilbert spaces. We show that these components are pathwise connected, too.
\end{abstract}

\section{INTRODUCTION}

The present paper has been inspired by a paper of Westphal and Frerking [6], in which the classical theory of monotone operators became a powerful and elegant tool for the investigation of the set of points of discontinuity of metric projections onto arbitrary closed subsets of Hilbert spaces. We prove that the image of certain connected sets by a maximal monotone operator is connected (Theorem 1). As an easy consequence of this general theorem we get a version of a result of Balaganskii [1], which provides the description of components (or, equivalently, pathwise connected components) of the set of those points, at which the metric projection to a closed set in Hilbert space is not continuous (Theorem 2). As a corollary we obtain the result from [6].

Throughout this paper, $X$ will denote a real Hilbert space. The open ball, closed ball, and sphere with center $x \in X$ and radius $r \geq 0$ will be denoted by $B(x ; r), \bar{B}(x ; r)$, and $S(x, r)(B(x ; 0)=\varnothing, \bar{B}(x ; 0)=S(x ; 0)=\{x\})$. The closed convex hull of a set $A \subset X$ will be denoted by $\overline{c o} A$. By $I$ we shall denote the identity mapping of $X ;[x, y]:=\{(1-t) x+t y ; 0 \leq t \leq 1\}$ for $x, y \in X$.

For a multivalued mapping $T: X \rightarrow 2^{X}$, the graph, range, and domain of $T$ (i.e., the set of $x$ with $T(x) \neq \varnothing$ ) will be denoted by $G(T), R(T)$, and $D(T)$. By $T / S$ we denote the restriction of $T$ on a set $S$, i.e.,

$$
w G(T / S)=G(T) \cap(S \times X)
$$

Received by the editors March 29, 1990.

1991 Mathematics Subject Classification. Primary 47H05, 41A65. 
We shall sometimes identify a point $y$ with the singleton $\{y\}$; hence $y \in T(x)$ and $T(x)=y$ will mean the same.

\section{MAIN RESUlT}

Let us recall the basic facts from the theory of monotone operators. We refer the reader to a classical book by Brézis [2], or to [5].

$T: X \rightarrow 2^{X}$ is a monotone operator if $\left\langle x-y, x^{*}-y^{*}\right\rangle \geq 0$ whenever $\left(x, x^{*}\right)$ and $\left(y, y^{*}\right)$ are elements of $G(T)$. A monotone operator $T$ is maximal if the set $G(T)$ is a maximal element (w.r.t. inclusion of sets) of the family of all graphs of monotone operators on $X$.

If $T$ is a maximal monotone operator on $X$ and $\lambda>0$, then $\overline{D(T)}$ is a convex set and any element $z$ of $X$ has a unique representation of the form

$$
z=J_{\lambda}(z)+\lambda T_{\lambda}(z) \text { with }\left(J_{\lambda}(z), T_{\lambda}(z)\right) \in G(T) .
$$

Moreover, the mappings $J_{\lambda}$ and $T_{\lambda}$ are Lipschitz with constants 1 and $\lambda^{-1}$, respectively. If in addition $z \in D(T)$, then

$$
\left\|J_{\lambda}(z)-z\right\| \leq \lambda \cdot \min \left\{\left\|z^{*}\right\| ; z^{*} \in T(z)\right\} .
$$

An important example of a maximal monotone operator represents the subdifferential $T=\partial f: X \rightarrow 2^{X}$ of a continuous convex function $f: X \rightarrow R$, defined by $\partial f(x)=\left\{x^{*} \in X ; f(y)-f(x) \geq\left\langle y-x, x^{*}\right\rangle\right.$ for every $\left.y \in X\right\}$. The Hahn-Banach theorem implies $D(\partial f)=X$.

Theorem 1. Let $T$ be a maximal monotone operator on a real Hilbert space $X$. If $S$ is a connected and relatively open subset of $\overline{D(T)}$, then the sets $G(T / S)$ and $T(S)$ are pathwise connected.

Proof. Let $\left(x, x^{*}\right)$ and $\left(y, y^{*}\right)$ be two points of $G(T / S)$. The convexity of $\overline{D(T)}$ implies that $S$ is pathwise connected [3, 6.3.10(a)]. Hence there exists a continuous $p:[0,1] \rightarrow S$ with $p(0)=x$ and $p(1)=y$. Denote $P=p([0,1])$. The compactness of $P$ implies that $\operatorname{dist}(P, \overline{D(T)}) \backslash S)=d>0$ and there exists a finite set $\left\{x_{1}, \cdots, x_{n}\right\} \subset D(T)$ such that $\operatorname{dist}\left(z,\left\{x_{1}, \cdots, x_{n}\right\}\right)<d / 3$ whenever $z \in P$. Choose arbitrarily $x_{i}^{*} \in T\left(x_{i}\right)$ for $i=1, \cdots, n$. Denote

$$
M=\max \left(\left\{\left\|x_{i}^{*}\right\| ; 1 \leq i \leq n\right\} \cup\left\{\left\|x^{*}\right\|,\left\|y^{*}\right\|\right\}\right), \quad z_{x}=x+\lambda x^{*}, \quad z_{y}=y+\lambda y^{*}
$$

where $\lambda>0$ is chosen in such a way that $\lambda M<d / 3$.

Let us define a continuous mapping $q:[-1,2] \rightarrow X$ by $q /[0,1]=p, q(-1)$ $=z_{x}, q(2)=z_{y}, q$ affine on $[-1,0]$ and on $[1,2]$. Denote $Q=q([-1,2])$ $=\left[z_{x}, x\right] \cup P \cup\left[y, z_{y}\right]$.

Let $z$ be an arbitrary element of $Q$. If $z \in P$, there exists an index $i$ such that $\left\|z-x_{i}\right\|<d / 3$, and then $\left\|J_{\lambda}(z)-z\right\| \leq\left\|J_{\lambda}(z)-J_{\lambda}\left(x_{i}\right)\right\|+\| J_{\lambda}\left(x_{i}\right)-$ $x_{i}\|+\| x_{i}-z\|\leq 2\| z-x_{i}\|+\lambda\| x_{i}^{*} \|<d$. If $z \in\left[z_{x}, x\right]$, then $\left\|J_{\lambda}(z)-x\right\| \leq$ $\left\|J_{\lambda}(z)-J_{\lambda}(x)\right\|+\left\|J_{\lambda}(x)-x\right\| \leq\|z-x\|+\lambda\left\|x^{*}\right\| \leq\left\|z_{x}-x\right\|+\lambda\left\|x^{*}\right\|<$ $2 d / 3<d$. Similarly, $\left\|J_{\lambda}(z)-y\right\|<d$ for any $z \in\left[y, z_{y}\right]$. Consequently, $\operatorname{dist}\left(J_{\lambda}(z), P\right)<d$ whenever $z \in Q$. This fact, together with $R\left(J_{\lambda}\right) \subset D(T)$, implies $J_{\lambda}(z) \in S$ for any $z \in Q$. Consequently, $J_{\lambda} \circ q$ is a path in $S$ with the initial point $J_{\lambda}\left(z_{x}\right)=x$ and the terminal point $J_{\lambda}\left(z_{y}\right)=y$. Moreover, the mapping $t \mapsto\left(J_{\lambda}(q(t)), T_{\lambda}(q(t))\right),-1 \leq t \leq 2$, is a path in $G(T / S)$ connecting $\left(x, x^{*}\right)$ and $\left(y, y^{*}\right)$. 


\section{CONNECTEDNESS STRUCTURE OF THE DISCONTINUITY SET FOR METRIC PROJECTIONS}

Let $F$ be a closed subset of $X$ and $x \in X$. We shall use the following notation.

$$
\begin{aligned}
& d_{F}(x)=\operatorname{dist}(x, F) \text { (distance function of } F \text { ), } \\
& P_{F}(x)=F \cap \bar{B}\left(x ; d_{F}(x)\right) \text { (metric projection of } F \text { ), } \\
& f_{F}(x)=\frac{1}{2}\|x\|^{2}-\frac{1}{2} d_{F}^{2}(x), \\
& C_{F}=\left\{x \in X ; P_{F}(x) \text { is a singleton and } P_{F} \text { is u.s.c. at } x\right\} \text { ("continuity } \\
& \text { points" of } \left.P_{F}\right) \text {. }
\end{aligned}
$$

For the following properties, the reader is referred to [6] and the references therein.

The metric projection $P_{F}$ is always monotone and the function $f_{F}$ is convex and continuous on $X$. Moreover, $G\left(P_{F}\right) \subset G\left(\partial f_{F}\right)$. The subdifferential $\partial f_{F}$ and the set $C_{F}$ have the following alternative representations.

$$
\begin{gathered}
\partial f_{F}(x)=\bigcap_{\varepsilon>0} \overline{\operatorname{co}}\left(F \cap \bar{B}\left(x ; d_{F}(x)+\varepsilon\right)\right), \\
C_{F}=\left\{x \in X ; P_{F}(x)=\partial f_{F}(x)\right\} .
\end{gathered}
$$

It follows from (1) that

$$
\partial f_{F}(x) \subset \bar{B}\left(x ; d_{F}(x)\right) \cap \overline{c o} F .
$$

It is easy to see that the $F \subset C_{F}$, because $P_{F}(x)=\partial f_{F}(x)=\{x\}$ for $x \in F$. (4) If $K \subset X$ is convex and closed, then the metric projection $P_{K}$ is singlevalued and continuous on $X$ (even nonexpansive, see, e.g., [4]).

Remark. The definition of $C_{F}$ implies that it is exactly the set of points of Fréchet differentiably of $f_{F}$, and therefore, since $X$ is an Asplund space, it is a dense $G_{\delta}$-subset of $X$ (cf. [4]).

Lemma 1. $\partial f_{F}(x) \cap S\left(x ; d_{F}(x)\right)=P_{F}(x)$ for each $x \in X$.

Proof. Only the inclusion $\partial f_{F}(x) \cap S\left(x ; d_{F}(x)\right) \subset P_{F}(x)$ for $x \notin F$ is not obvious. Without any loss of generality we can suppose $x=0$. Denote $r=$ $d_{F}(0)$.

Let $z \in S(0 ; r) \backslash P_{F}(0)$. There exists a $\delta>0$ such that $B(z ; \delta) \cap F=\varnothing$. Hence, by (1)

$$
\partial f_{F}(0) \subset \bigcap_{\varepsilon>0} \overline{\operatorname{co}}(\bar{B}(0 ; r+\varepsilon) \backslash B(z ; \delta)) .
$$

Choose an $\varepsilon>0$ such that $\varepsilon(2 r+\varepsilon) \leq \delta^{2}$. Then for any $y \in \bar{B}(0 ; r+\varepsilon) \backslash B(z ; \delta)$ we have

$$
\langle z, y\rangle=\frac{1}{2}\left(\|z\|^{2}+\|y\|^{2}-\|z-y\|^{2}\right) \leq \frac{1}{2}\left(r^{2}+(r+\varepsilon)^{2}-\delta^{2}\right) \leq r^{2}-\frac{1}{2} \delta^{2} .
$$

Consequently, $\langle z, y\rangle \leq r^{2}-\delta^{2} / 2$ for any $y \in \overline{\operatorname{co}}(\bar{B}(0 ; r+\varepsilon) \backslash B(z ; \delta))$. But this, together with $(5)$ and $\langle z, z\rangle=r^{2}$, implies $z \notin \partial f_{F}(0)$.

Lemma 2. Let $S$ be a component of the set $\overline{\operatorname{co}} F \backslash F$. If $\partial f_{F}(x) \cap S \neq \varnothing$ then $P_{\overline{\text { co } F}}(x) \in S$. 
Proof. Obviously $d_{\overline{\mathrm{co}} F}(x) \leq d_{F}(x)$. (4) implies $d_{\overline{\mathrm{co}} F}(x)<d_{F}(x)$ and $P_{\overline{\mathrm{co}} F}(x)$ $\notin F$ (otherwise $P_{\overline{\mathrm{co}} F}(x)=P_{F}(x)=\partial f_{F}(x) \subset F$ ). Consequently

$$
\begin{aligned}
P_{\overline{\mathrm{co}} F}(x) & =\bar{B}\left(x ; d_{\overline{\mathrm{co}} F}(x)\right) \cap \overline{\mathrm{co}} F=\bar{B}\left(x ; d_{\overline{\mathrm{co}} F}(x)\right) \cap(\overline{\mathrm{co}} F \backslash F) \\
& \subset B\left(x ; d_{F}(x)\right) \cap(\overline{\mathrm{co}} F \backslash F)=B\left(x ; d_{F}(x)\right) \cap \overline{\mathrm{co}} F=: A,
\end{aligned}
$$

where $A \subset B\left(x ; d_{F}(x)\right)$ is a connected (in fact convex) subset of $\overline{c o} F \backslash F$. But by (3) and Lemma $1, \partial f_{F}(x) \cap S \subset A$. Therefore $A \subset S$.

The following theorem is due in main to Balaganskii [1], whose proof is rather long and complicated. We obtain this result as an almost immediate consequence of Theorem 1 and the two simple lemmas above.

Theorem 2. Let $F$ be a closed subset of a real Hilbert space $X$. Let $\mathscr{S}$ and $\mathscr{M}$ be the families of all components of the set $\overline{c o} F \backslash F$ and of the set $X \backslash C_{F}$, respectively. Then the following holds.

(a) $X \backslash C_{F}=\left\{x \in X ; \partial f_{F}(x) \cap(\overline{\mathrm{co}} F \backslash F) \neq \varnothing\right\}$.

(b) The mapping $S \mapsto M_{S}=\left\{x \in X ; \partial f_{F}(x) \cap S \neq \varnothing\right\}$ is a bijection of $\mathscr{S}$ onto $\mathscr{M}$. Moreover, $M_{S}=\left(X \backslash C_{F}\right) \cap P_{\overline{\mathrm{co}} F}^{-1}(S)$.

(c) The inverse of the bijection is the mapping $M \mapsto S_{M}$ which can be defined in the following way: for $M \in \mathscr{M}$ choose an arbitrary $x \in M$ and denote by $S_{M}$ the element of $\mathscr{S}$ which contains the point $P_{\overline{\mathrm{co}} F}(x)$.

(d) The elements of $\mathscr{M}$ and of $\mathscr{S}$ are pathwise connected and relatively open in $X \backslash C_{F}$ and in $\overline{\mathrm{co}} F \backslash F$, respectively.

Proof. (a) follows easily from (3), Lemma 1, from the convexity of $\partial f_{F}(x)$ for $x \in X$ and from the fact that $X$ is strictly convex. Each $S \in \mathscr{S}$, being a component of a relatively open subset of a convex set, is pathwise connected and relatively open in $\overline{c o} F \backslash F$ [3]. Consider the maximal monotone operator $T=\left(\partial f_{F}\right)^{-1}$. By (3) and by $T / F=I / F$, we have $F \subset R\left(\partial f_{F}\right)=D(T) \subset$ $\overline{\text { co }} F$. The convexity of $\overline{D(T)}$ implies $\overline{D(T)}=\overline{\text { co } F}$. By Theorem 1 , the sets $M_{S}=T(S)$ are pathwise connected. If $M \in \mathscr{M}$ is such that $M_{S} \subset M$, then by (a), Lemma 2 and (4), the set $P_{\overline{\mathrm{co}} F}(M)$ is connected and it is contained in a component of $\overline{c o} F \backslash F$, namely in $S$. From this we deduce $M=M_{S}$. This proves (b) and (c). Now, for any $M \in \mathscr{M}$ we have $M=\left(X \backslash C_{F}\right) \cap P_{\overline{\operatorname{co}} F}^{-1}(S)$, and hence $M$ is relatively open in $X \backslash C_{F}$ by (4).

Corollaries I [6]. If $x$ is a nonisolated element of $X \backslash C_{F}$, then there exists a nontrivial path in $X \backslash C_{F}$ containing $x$.

II. If $X \backslash C_{F}$ is not an isolated set, then $\operatorname{card}\left(X \backslash C_{F}\right) \geq \operatorname{card}([0,1])$.

III. The set $X \backslash C_{F}$ has at most dens $(X)$ components (dens $(X)$ is the smallest cardinal number of the form $\operatorname{card}(A)$ where $A$ is a dense subset of $X$; [3]). In particular, $X \backslash C_{F}$ has at most countably many components if $X$ is separable.

IV. If $X \backslash F$ is bounded and connected, then the set $X \backslash C_{F}$ is pathwise connected.

Proof. I. (d) implies the existence of a point $y \in X \backslash C_{F}$, distinct from $x$, such that $x$ and $y$ are elements of the same component of $X \backslash C_{F}$. Hence $x$ and $y$ can be connected by a path in $X \backslash C_{F}$. 
II follows immediately from I.

III is a consequence of (b).

IV In this case, $\overline{\operatorname{co}} F=X$. Apply (b).

\section{AKNOWLEDGMENT}

This paper was written during the author's fellowship at G.N.A.F.A. of Consiglio Nazionale delle Ricerche, Firenze, Italy.

\section{REFERENCES}

1. V. S. Balaganskii, On the connection between approximative and geometrical properties of sets, Approximation in Concrete and Abstract Banach Spaces, Akad. Nauk SSSR, Ural'skii Naučnyi Centr, pp. 46-53, 1987. (Russian)

2. H. Brézis, Opérateurs maximaux monotones et semi-groupes de contractions dans les espaces de Hilbert, North-Holland Math. Studies, no. 5, Amsterdam, 1973.

3. R. Engelking, General topology, Monografie Matematyczne 60, Warszawa, 1977.

4. J. R. Giles, Convex analysis with application in the differentiation of convex functions, Research Notes in Math., no. 58, Pitman, Boston 1982.

5. D. Pascali and S. Sburlan, Nonlinear mappings of monotone type, Bucuresti and Alphen aan den Rijn, 1978.

6. U. Westphal and J. Frerking, On a property of metric projections onto closed subsets of Hilbert spaces, Proc. Amer. Math. Soc. 105 (1989), 644-651.

Via S. Vitale 4, 40125 Bologna, Italy 